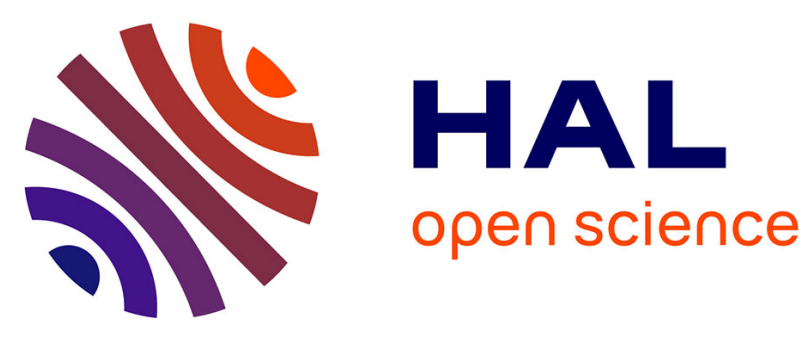

\title{
AFNOR validation of Premi Test, a microbiological-based screening tube-test for the detection of antimicrobial residues in animal muscle tissue.
}

Valerie Gaudin, Murielle Juhel-Gaugain, Jean-Pierre Morétain, Pascal Sanders

\section{To cite this version:}

Valerie Gaudin, Murielle Juhel-Gaugain, Jean-Pierre Morétain, Pascal Sanders. AFNOR validation of Premi Test, a microbiological-based screening tube-test for the detection of antimicrobial residues in animal muscle tissue.. Food Addit Contam Part A Chem Anal Control Expo Risk Assess, 2008, 25 (12), pp.1451-1464. 10.1080/02652030802429088 . hal-00606192

\section{HAL Id: hal-00606192 \\ https://hal-anses.archives-ouvertes.fr/hal-00606192}

Submitted on 5 Jul 2011

HAL is a multi-disciplinary open access archive for the deposit and dissemination of scientific research documents, whether they are published or not. The documents may come from teaching and research institutions in France or abroad, or from public or private research centers.
L'archive ouverte pluridisciplinaire $\mathbf{H A L}$, est destinée au dépôt et à la diffusion de documents scientifiques de niveau recherche, publiés ou non, émanant des établissements d'enseignement et de recherche français ou étrangers, des laboratoires publics ou privés. 


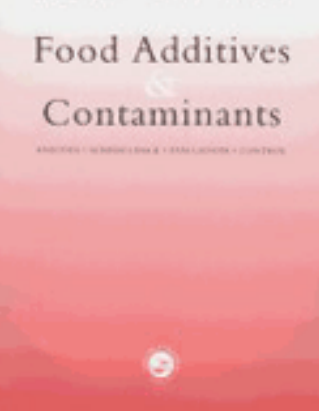

\section{AFNOR validation of PremiTest, a microbiological-based screening tube test for the detection of antimicrobial residues, in muscles from different animal origins}

\begin{tabular}{|r|l|}
\hline Journal: & Food Additives and Contaminants \\
\hline Manuscript ID: & TFAC-2008-016.R1 \\
\hline Manuscript Type: & Original Research Paper \\
\hline Author: & 30 -Jul-2008 \\
\hline Complete List of Authors: & $\begin{array}{l}\text { Gaudin, Valerie; AFSSA, LERMVD, CRL for antimicrobial residues in } \\
\text { food } \\
\text { Gaugain-Juhel, Murielle; AFSSA, LERMVD; AFSSA, LERMVD, CRL for } \\
\text { antimicrobial residues in food } \\
\text { Moretain, Jean Pierre; Agence Francaise de Securite Sanitaire des } \\
\text { Aliments-CRL for antimicrobial residues in food, Laboratory for the } \\
\text { research and study of veterinary medicinal products and } \\
\text { disinfectants } \\
\text { Sanders, Pascal; AFSSA, LERMVD }\end{array}$ \\
\hline Methods/Techniques: & $\begin{array}{l}\text { In-house validation, Inter-laboratory validation, Screening - } \\
\text { microbial screening, Screening assays }\end{array}$ \\
\hline Additives/Contaminants: & $\begin{array}{l}\text { Veterinary drug residues - antibiotics, Veterinary drug residues - } \\
\text { antimicrobials }\end{array}$ \\
\hline Food Types: & Animal products - meat, Meat \\
\hline & \\
\hline
\end{tabular}

\section{SCHOLARONE \\ Manuscripts}


AFNOR validation of Premi ${ }^{\circledR} T e s t$, a microbiological-based screening tube test

2 for the detection of antimicrobial residues, in muscles from different animal

3 origins

4

5

6 Valérie Gaudin*, Murielle Juhel-Gaugain, Jean-Pierre Morétain, Pascal Sanders

7

8 Community Reference Laboratory

9 AFSSA Fougères - LERMVD

10 La Haute Marche - BP 90203

1135302 FOUGERES Cedex

12 France

16 *Address for correspondence : E-mail: v.gaudin@fougeres.afssa.fr 


\section{Abstract}

Premi®Test contains viable spores of a strain of Bacillus stearothermophilus which is sensitive to antimicrobial residues such as beta-lactams, tetracyclines, macrolides, and sulphonamides etc. The growth of the strain is inhibited by the presence of antimicrobial residues in muscle samples. Premi®Test was validated according to AFNOR rules (French Association for Normalisation). The AFNOR validation was based on the comparison of a reference method [French Official method (Four Plate Test) and the STAR protocol (5 plate test)] with the alternative method (PremißTest). A preliminary study was conducted in an expert laboratory (Community Reference Laboratory $\mathrm{CRL}$ ) on both spiked and incurred samples (field samples). Several method performance criteria (sensitivity, specificity, relative accuracy) were estimated and are discussed, in addition to detection capabilities. Adequate agreement was found between alternative methods and the reference method. However, Premi ${ }^{\circledR}$ Test was more sensitive to beta-lactams and sulphonamides than the FPT. Subsequently, a collaborative study with 11 laboratories was organised by the CRL. Blank and spiked meat juice samples were sent to participants. The expert laboratory $(C R L)$ statistically analysed the results. It was concluded that Premi ${ }^{\circledR}$ Test could be used for the routine determination of antimicrobial residues in muscle of different animal origin with acceptable analytical performance. The detection capabilities of Premi®Test for beta-lactams (amoxicillin, ceftiofur), one macrolide (tylosin) and tetracycline were at the level of the respective Maximum Residue Limits (MRL) in muscle samples or even lower.

Keywords: Validation, Premi®test, screening, antimicrobial residues, muscle, collaborative study, routine analysis 


\section{Introduction}

Animal treatment can lead to the presence of residues in food of animal origin. The presence of antimicrobial residues could lead to human safety problems such as allergies or toxicity when foodstuffs containing residues enter the food chain. Different families of antimicrobial residues are concerned: beta-lactams, sulfonamides, macrolides, tetracyclines, quinolones, etc. For these reasons, Maximum Residue Limits (MRL) were set for many antimicrobial residues to protect consumer safety (EEC Directive 2377/90 and amendments). Different methods were developed for the detection of antimicrobial residues in food of animal origin. These screening methods are usually microbiological methods which are based on the inhibition of bacterial growth by antimicrobial residues. Microbiological plate tests generally give results in 18 to 24 hours. However, these home-made methods require skilled technicians, with a specific training. Microbiological based tests are interesting at the screening step because they are able to detect a wide range of antimicrobial residues (several families of antimicrobials are detectable with one single method). The levels of detection of these methods are generally satisfactory with respect to European regulations (EEC, 1990) for beta-lactams, tetracyclines and macrolides (detection capabilities lower or equal to the MRL). The best detected class of compound is generally the beta-lactam family. The detection capabilities of sulphonamides are very variable depending on the sulphonamide structure. The least detected antibiotics are generally some sulphonamides, aminoglycosides and quinolones by microbiological based tests. None of the existing microbiological screening methods are able to detect all the MRL substances in animal matrices. Therefore, some countries implemented other kind of methods in parallel to look for these antimicrobials specifically. In France, the official method for the control of muscle samples is the Four Plate Test (FPT) (Bogaerts and Wolf 1980). In the past, some commercial tube tests already exist but only for the analysis of milk, like Delvotest ${ }^{\circledR}$ and since a few years COPAN ${ }^{\circledR}$ test. These tests are based on the detection of growth by production of acid, visible by a colour change of the test medium ( $\mathrm{pH}$ indicator). More recently, the Premi ${ }^{\circledR} T e s t$, a commercial growth inhibitor test, was developed for the detection 
of antimicrobial residues in muscle by DSM (DSM Food Specialities R\&D, Delft, the Netherlands). This test is based on the inhibition of the strain Bacillus stearothermophilus. Premi®Test allows to detect antimicrobial residues in muscle in less than 4 hours (Fabre 2003; Stead. 2004; Fabre et al. 2004)).

Over the last few years, several evaluations of the Premi®Test have already been published, based on spiked meat juice samples (Reybroeck 2000a) or incurred poultry muscles (Reybroeck 2000b). Premi®Test has generally been compared to a reference method. Some studies were focused on a family of antimicrobials like beta-lactams, using spiked meat juice samples and incurred poultry muscle samples (Popelka et al. 2005) or tetracyclines (Okerman et al. 2004). The detection limits of different antimicrobials, calculated in spiked juice samples, were very near in the different studies. The global conclusions of the different studies were that Premi®Test is not suited for the detection of tetracyclines at MRL level in spiked and incurred samples. Moreover, Premi ${ }^{\circledR}$ Test was very sensitive for the screening of beta-lactams, more than usual microbiological plate tests. PremißTest is suited for the detection of beta-lactams and sulphonamides, at MRL level or even below MRL.

The AFNOR validation is based on the comparison between a reference method and an alternative method. In the present study, the Premi ${ }^{\circledR} T e s t$, the alternative method, was compared with 2 other microbiological methods: the French Official method (Four Plate Test) which was the reference method and the STAR protocol (the CRL protocol) (Gaudin et al. 2004). Afterwards, an interlaboratory study was organised, where only the Premi $囚$ Test has been used to analyse spiked meat juice samples. This paper presents the results of preliminary and collaborative studies.

\section{Materials and methods}




\section{Presentation of the methods}

Principle of the kit. The PremißTest allows to detect antimicrobial residues in fresh meat, kidneys, fish and eggs (Arts et al. 2000). The Premi®Test is based on the growth inhibition of Bacillus stearothermophilus. Standardized spores are included in a medium, with selected nutrients. The meat juice was put on ready-to-use tubes. After 20 min of pre-diffusion at room temperature, the meat juice was removed by three washing steps. Finally, the ampoule was incubated during approximately 3 hours at $64^{\circ} \mathrm{C}$. The reading of the "yes/no" result was based on a colour comparison. Without antimicrobials, the spores germinated and developed, involving the acidification of the medium and a change of colour (yellow). Conversely, in the presence of antimicrobials, the bacterial growth was inhibited. A purple colour indicated the presence of antibiotics, at or above the detection limit of the test. Doubtful and positive samples were confirmed by the multi-residue LC/MS-MS method described below. This broad spectrum test makes it possible to detect a great number of antimicrobials usually used, in less than 4 hours, on muscle juice samples (extracted by pressing a piece of meat).

Principle of the Four Plate Test (FPT). The Four Plate Test is the French Official method for the control of muscle samples (Bogaerts and Wolf 1980). A microorganism sensitive to antibacterial substances is inoculated into an agar medium in a Petri dish. The following test organisms are used: Bacillus subtilis BGA (reference 10649, Merck) (in test agar $\mathrm{pH} 6$, Merck), Bacillus subtilis BGA (reference 10649, Merck) (in test agar pH 8, Merck), Bacillus subtilis BGA (reference 10649, Merck) (in agar ASS pH 7.4, Merck) and Kocuria varians (ATCC 9341, Pasteur Institute) (in test agar $\mathrm{pH} 8$, Merck). Slices of frozen muscle were placed on the surface of the inoculated medium, and then incubated at the optimal temperature for growth of the test organism. After diffusion, the presence of antibacterial substance should produce an inhibition zone around the sample by inhibiting the growth of the test organism. 
Principle of the STAR protocol and Bacillus cereus. The STAR protocol is the CRL method (Gaudin et al. 2004) which was developed to improve the performance of the Four Plate Test. The detection principle is the same as Four Plate Test. The following test organisms are used: Bacillus subtilis BGA (reference 10649, Merck) (Antibiotic medium II at pH 8.0, Difco), Kocuria varians ex. Micrococcus luteus (ATCC 9341, Pasteur Institute) (in test agar at $\mathrm{pH}$ 8, Merck), Bacillus cereus Bc6 (ATCC 11778, Pasteur Institute) (in test agar at pH 6, Merck), Escherichia coli (ATCC 11303, Pasteur Institute) (in test agar at pH 8, Merck), Bacillus stearothermophilus (ATCC 10149, Merck) (in DST (Diagnostic Sensitive Test) medium, Oxoid). The plate Bacillus cereus which was used by the field laboratories at the $3^{\text {rd }}$ step of the preliminary study is the plate Bc6 of the STAR protocol, which is selective for the detection of the tetracyclines' family. Slices of muscle samples of $2 \mathrm{~mm}$ in thickness are cut in frozen muscle and put on the plates.

LC/MS-MS method. A multi-residue LC/MS-MS method was developed for the screening of antimicrobials in meat. The principle is based on two different extractions : one with trichloroacetic acid (TCA) allowing the detection of tetracyclines, aminoglycosides and quinolones, the second with acetonitrile (ACN) allowing the detection of penicillins, cephalosporins, macrolides and sulfonamides. TCA extracts are directly injected after ultracentrifugation and filtration. ACN extracts are evaporated and the residue is then dissolved in $0.6 \mathrm{ml}$ of ammonium acetate before filtration and injection. Two different gradients with pentafluoropropionic acid and ACN are used for the LC analyses. LC/MS-MS is used with a Multi Reaction Monitoring Mode (MRM) and two MRM transitions are monitored for each compound. The identification of the detected compounds is based on the retention time and the presence of the two specific transitions. The quantitative determination was carried out by using calibration curves obtained with spiked samples at $0.5 \mathrm{MRL}, 1 \mathrm{MRL}$ and 1.5 MRL levels. Internal standard were used for the quantification. All the 50 monitored compounds except some aminoglycosides were detected at a level below the MRL. 


\section{Principle of AFNOR (French Association for Normalisation) validation}

An alternative method should be compared with a reference method. A reference method could be a standardized method when it exists, an official method, or a widely known and used method, taken in reference. An alternative method is a commercial test allowing to analyze, for a category of products given, the same analytes as that measured by the reference method, but which presents moreover, one or more criteria following: speed of analysis, easiness of execution and/or automation, analytical performances (limit of detection, specificity, etc). A specific guide for validation of alternative methods in the field of detection of antimicrobial residues in foodstuffs of animal origin was edited (Anon. 2005a). It defined the requirements relating to the organisation of preliminary and collaborative studies, carried out by one expert laboratory. This document established the general principle as well as the technical protocol for the validation of alternative methods in the field of detection of antimicrobial residues in foodstuffs of animal origin.

\section{Preliminary study}

The preliminary step has been divided in 3 parts.

Step 1. Analysis of blank and spiked meat juice samples. Porcine muscles came out of practice. The absence of antimicrobial substances in these pig samples, assumed as blank materials, was checked with the FPT and the STAR protocol. The negative results confirmed that these pig samples could be used to prepare blank meat juices.

Muscle samples were pressed with garlic press. Afterwards, meat juice samples were spiked with known concentrations of 6 different antimicrobials: sulfamethazine (sulphonamides), oxytetracycline (tetracyclines), tylosin (macrolides), amoxicillin (penicillins), ceftiofur (cephalosporins) and gentamycine (aminoglycosides). Each antimicrobial was spiked at 3 concentrations: sulfamethazine $\left(50,100,200 \mu \mathrm{g} \mathrm{kg}^{-1}\right)$, oxytetracycline $\left(50,100,200 \mu \mathrm{g} \mathrm{kg}^{-1}\right)$, tylosin $\left(50,100,200 \mu \mathrm{g} \mathrm{kg}^{-1}\right)$, amoxicillin $\left(25,50,100 \mu \mathrm{g} \mathrm{kg}^{-1}\right)$, ceftiofur $(100,200,400 \mu \mathrm{g} \mathrm{kg}$ $\left.{ }^{1}\right)$ and gentamycine $\left(50,100,200 \mu \mathrm{g} \mathrm{kg}^{-1}\right)$. 
1785 aliquots (5 combinations sample/antimicrobial/concentration) were prepared "blind to the 179 technician" and analysed in duplicate only with the Premi®Test. The aliquots were stored in freezer (approximately $-20^{\circ} \mathrm{C}$ ) before analyses. Blank meat juice and spiked meat juice samples were analysed in duplicate only with the Premi®Test.

Step 2. Treatment of animals and analysis of incurred porcine muscle samples. Two untreated pigs were slaughtered before the treatments of 3 other pigs. These pigs were supplied by a farm guaranteeing that the pigs did not receive antimicrobial treatments before. Moreover, the absence of antimicrobials in muscle samples was checked with the FPT and the STAR protocol.

Three pigs were treated: one with tylosin, one with amoxicillin and one with a mXture of oxytetracycline and sulfadimethoxine and slaughtered. Each material was analysed by a multi-residue LC/MS-MS method to quantify antimicrobials. The following concentrations were obtained: tylosin $750.9 \pm 76.1 \mu^{\prime g k g}{ }^{-1}$ (material 1), amoxicillin $269.5 \pm 18.0 \mu \mathrm{gkg}^{-1}$ (material 2), oxytetracycline $764.9 \pm 44.8 \mu \mathrm{gg}^{-1}$ and sulfadimethoxine $151.1 \pm 13.2 \mu \mathrm{gkg}^{-1}$ (material 3).

Afterwards, 5 pieces of each material (blank and treated animals) of approximately $20 \mathrm{~g}$ were (5 combinations sample/antimicrobial/concentration) prepared "blind to the technician" and analysed in duplicate with 3 methods: Premi®Test, STAR protocol and FPT. These samples were stored in freezer at approximately $-20^{\circ} \mathrm{C}$ before analyses.

Step 3. Analysis of field samples. This step allowed to compare PremißTest, STAR protocol and FPT, on "naturally" incurred samples, for a wide number of antimicrobials and matrices of different origins (e.g. different species).The samples came from a pilot study concerning the implementation of a new screening method of the antimicrobial residues in meat (Anon. 2005b). The samples were sent regularly to laboratories from the French veterinary services (official control). SX field veterinary laboratories (LVD or "Laboratoire Vétérinaire Départemental" in French) were trained to the implementation of the Premi®Test and one 
206 plate Bacillus cereus (detection of tetracyclines) (STAR protocol) and analyzed 1427 field 207 samples, over a period of 4 months. All their positive samples (with Premi®Test and/or 208 Bacillus cereus) were sent frozen to the CRL, every 15 days. All the samples characteristics 209 (species, reception date, analysis date, sending date, ...etc...), as well as the results 210 obtained with Premi®Test and Bacillus cereus, were also sent to the CRL. All these samples 211 coming from the field laboratories where stored in the freezer at their arrival in our laboratory. 212 Then, blind analyses were carried out at the CRL with the Premi®Test, the FPT, and the 213 STAR protocol including the Bacillus cereus plate. Finally, the positive samples with at least 214 one of these methods were analyzed by the multi-residue LC/MS-MS method (systematic 215 screening, then identification and quantification). The doubtful samples with PremißTest 216 were also tested by LC/MS-MS.

Interlaboratory study

11 laboratories have been contacted to participate to this inter-laboratory study, including the 6 field laboratories which had already participated to the step 3 of the preliminary study. When an AFNOR validation is implemented, an expert laboratory is designated by the AFNOR Technical Office. The expert laboratory performed the intra-laboratory validation and organised the inter-laboratory study. Moreover, during the inter-laboratory study, the results of the expert laboratory are not included with all the participants' results, but these results are considered as the reference results (to be obtained by the participants). in this case, the Community Reference Laboratory was considered as the expert laboratory.

Spiked muscle juice samples were used for the interlaboratory study, instead of incurred samples, for several reasons: Firstly, it is very difficult to prepare raw muscle with a target concentration of each antimicrobial because of the individual variability of animals' pharmacokinetic (depletion of the antibiotic in tissues). Moreover, the use of raw pieces of meat did not allow to mince meat to adjust the concentration. Secondly, the study was based on several animal species. Therefore, the treatment should have been performed in each 
specie. This would have been very expensive, time consuming and impossible to be implemented in our laboratory. Thirdly, when producing incurred muscles and sending raw muscle samples (pieces of meat), it is very difficult to ensure the homogeneity of the samples, which is a basic condition when organising a collaborative study.

Preparation of the materials. Porcine, bovine and chicken muscles were pressed. Blank meat juice samples (negative controls and blank unknown samples) were prepared. Furthermore, spiked samples were prepared with 4 different antibiotics, at 3 concentrations Oxytetracycline and ceftiofur were added to porcine meat juice samples, sulfamethazine to bovine meat juice samples and finally tylosin to chicken meat juice samples Table I presents the content of the 16 combinations.

\section{"[Insert Table I about here]"}

A stability study was carried out on materials before sending of the samples and over the period of analyses by the participants. The samples were analysed with the Premi®Test at the CRL. Stability of materials was proved over the period of analyses. A random codification of the materials was performed. Each laboratory was identified by a code (from A to P).

Sending of the materials. One negative control from each species was sent and one positive control containing penicillin $\mathrm{G}$ at $10 \mu \mathrm{g} \mathrm{kg}^{-1}$. 32 different frozen meat juice samples (16 materials in blind duplicate: spiked and blank samples) were sent under frozen conditions (dried ice) to the participants, in order to ensure stability of matrix and analyte. The samples were stored in freezer at their arrival. Eleven laboratories received with their parcel instructions and a results form. The participants had no information about the antibiotics contained in the materials.

Because it is recommended to test one negative control from each analysed species, each laboratory received a table indicating the species of origin of each sample, according to sample code (1 to 32 ), in order to compare the results of each sample with the negative 
control of the corresponding species. All analyses were carried out in blind duplicate (2 different series of analyses), with Premi®Test only. The analyses were performed within one week maximum after receiving the samples. A negative control for each species, a positive sample (spiked with penicillin $\mathrm{G}$ at $10 \mu \mathrm{g} \mathrm{kg}-1$ ) and coded materials were analysed. All the results were returned rapidly and compiled at the CRL.

\section{Results and discussion}

\section{Preliminary study}

Step 1. Analysis of blank and spiked meat juice samples. Among the 20 blank meat juice samples analysed in duplicate, only one meat juice was positive twice and 4 other meat juices were "doubtful" on one of the two repetitions and negative on the other. 15 meat juices were negative twice. The false positive rate is the number of positive results for blank samples (free of antibiotic substances) divided by the total number of positive samples (the same number plus the number of contaminated samples showing positive results) and multiplied by $100.18 \%\left(=6 /(6+27)^{\star} 100\right)$ of false positive results was observed. The result was satisfactory because the false positive rate should be minimal for a screening method, since the samples declared positive should be confirmed by a physicochemical method for identification and quantification.

The detection limit corresponded to the lowest concentration which gave a positive or a doubtful result for each of the 5 replicates. Table II presents the determination of limits of detection of Premi®Test for the 6 antimicrobials tested.

$$
\text { "[Insert Table II about here]" }
$$

The study of spiked pig meat juice samples showed that, for 5 molecules belonging to 4 different classes of antibiotics, the detection limit of Premi®Test was at the level of one or two MRL maximum. For only one antibiotic (gentamicin), the detection limit was higher than 2 times the MRL ( $40 \%$ of positive results at $2 x M R L)$. The false negative rate corresponds to 
290 the number of negative results obtained for contaminated samples (spiked samples) divided 291 by the total number of negative samples (the same number plus the number of blank 292 samples giving a negative result) and multiplied by 100 . The false negative rate calculated at $2931^{*} \mathrm{MRL}$ was equal to $22 \%(=10 /(10+34))$. However, the false negative rate calculated at 294 twice the MRL was equal to $8 \%(=3 /(3+34))$.

Step 2. Treatment of animals and analysis of incurred porcine muscle samples. The concentrations of naturally incurred samples were much higher than the respective MRL of the 4 antimicrobials (from 1.5 to 7.6 times the MRL). However, the analyses of incurred materials with known antimicrobial concentrations was of great interest. Table III summarises the results of step 2 .

The data exploitation was carried out according to the reference document of AFNOR validation (Anon. 2005a). The exploitation is based on the comparison of 2 methods: the reference method and the alternative method. The FPT was set as the reference method and the Premi®Test is the alternative method.

Three different parameters were calculated which allowed to compare reference and alternative method: relative accuracy $\left(A C=(P A+N A) / N^{*} 100 \%\right)$, relative specificity $(S P=$ $\left.(\mathrm{NA} / \mathrm{N}-)^{*} 100 \%\right)$, relative sensitivity $\left(\mathrm{SE}=(\mathrm{PA} / \mathrm{N}+)^{*} 100 \%\right)$, where: $\mathrm{NA}$ is the negative agreement (negative result obtained with both methods), PA: the positive agreement (positive result obtained with both methods), ND: the negative discrepancy (positive result obtained with reference method and negative result with alternative test), PD: the positive discrepancy (negative result obtained with reference method and positive result with alternative test); $N=N A+P A+P D+N D$ : total number of samples; $N$ - is the total number of negative samples obtained with the reference method (NA + PD); $N+$ is the total number of positive samples obtained with the reference method (PA $+N D)$. 
318 The relative accuracy, the relative specificity and the relative sensitivity were calculated as $31970 \%, 42.9 \%$ and $84.6 \%$ respectively. Therefore, the relative accuracy and specificity were 320 quite satisfactory. The low value of relative specificity could be explained because the sensitivity of the FPT was sometimes insufficient for certain antibiotics.

In conclusion, FPT and Premi®Test gave concordant results when looking at the performance characteristics (relative accuracy, the relative specificity and the relative sensitivity). However, at this step, the false negative and the false positive rates were lower for the PremißTest (both $0 \%$ ) than for the FPT (33 and $40 \%$ respectively).

Step 3. Analysis of field samples. 1427 field incurred samples, originated from 6 French field veterinary laboratories, were analyzed implementing PremißTest and Bacillus cereus plate. 1325 samples were detected negative and 102 samples doubtful (36) and positive (66) with the PremißTest. other samples were found positive only on plate Bc6. Finally, the presence of a tetracycline

Furthermore, among the 1325 negative results, 10 samples were detected positive with the Bacillus cereus plate. Therefore, 112 muscle samples were sent to the $C R L$ to be confirmed. The correlation between the Premi®Test results of LVD and AFSSA was studied (Table IV). $76 \%$ of the samples $(78 / 102)$ found positive or doubtful with Premi®Test in the LVD, were found positive or doubtful with Premi®Test at the CRL. 24 samples were positive or doubtful at the LVD and negative at AFSSA. It could be due either to false positive results of the field laboratories, or to antibiotic instability between the 2 analyses, although the best storage and transport conditions have been strictly respected. Among the 10 samples negative with Premi®Test and positive with Bacillus cereus in the field laboratories, 6 were found positive or doubtful with Premi®Test at the CRL and confirmed positive with Bacillus cereus. The 4 was confirmed by LC/MS-MS in 9 of these samples. Therefore, Bacillus cereus plate (one 
plate of the STAR protocol, which is selective for the detection of tetracyclines) is more sensitive for tetracyclines than Premi®Test.

"[Insert Table IV about here]"

The false positive rate in field laboratories was sometimes high, mainly at the beginning of the study. Afterwards, with the reading experience, most of the positive results with Premi®Test at the field laboratories were confirmed positive with Premi®Test at the CRL.

Many animal species were studied in step 3 because samples were from field laboratories origin, while the 2 first steps were based only on pig muscle. The distribution of the samples in the different species is presented (Table IV).

Confirmatory rate of LC/MS-MS method (number of samples really containing antimicrobial residues divided by the number of tested samples (positive screening) multiplied by 100) was equal to $41 \%(25 / 61)$. The confirmatory rate varied between species. There were more false positive results in some species $(37 \%$ for bovine samples instead of $75 \%$ for poultry samples).

The results of the comparative analysis between the alternative method PremißTest and the reference method FPT are presented in Table V.

$$
\text { "[Insert Table V about here]" }
$$

When the data analysis was based only on the 112 samples reanalysed in the expert laboratory, relative accuracy (33.9\%) and relative specificity (33.4\%) were very low. Only relative sensitivity $(70 \%)$ was satisfactory, as in the first step of the validation study. However, in this case, Premi®Test negative samples (1315 negative samples / 1427 analyzed samples) obtained in the field laboratories were not taken into account. Only the positive samples in the field laboratories were reanalysed at the CRL with Premi®Test and FPT. None of the 1315 negative samples was analysed with the FPT. By experience (a 
373 previous study in 2005) (data not shown), all the samples which were declared negative with 374 Premi®Test and negative with Bacillus cereus were also all negative with the FPT (28 375 samples). Therefore it was assumed that the 1315 samples, found negative with both 376 PremißTest and Bacillus cereus, would have also been negative with the FPT. In this case, 377 the agreement (relative accuracy $94.8 \%$, relative specificity $95 \%$ ) between the results of 378 PremißTest and FPT was very satisfactory. The 2 methods were declared different by a 379 statistical test, because of a high rate of positive deviation (Premi®Test + / FPT -) which may 380 be caused partially by false positive results of the Premi®Test and/or by the lack of sensitivity of the reference method towards some antimicrobial residues.

The agreement (relative accuracy) between the results of Premi®Test and STAR was equal to $42.99 \%$, the relative specificity $31.3 \%$ and the relative sensitivity $71.9 \%$. Therefore, it is quite similar to the comparison of FPT with Premi®Test. The agreement between the FPT and the STAR protocol was higher (70\%) (data not shown). Only one sample was negative with the STAR protocol and positive with the FPT. The absence of antimicrobial residues in this sample was confirmed by LC/MS-MS.

The results obtained in the field laboratories, and then at the $C R L$, with the Premi®Test, the FPT, the STAR protocol and the confirmatory method by LC/MS-MS are compared (Table VI). The number of positive results with the Premi®Test (78) was much higher than the number of positive with the multi-plate tests (FPT 10; STAR 31 samples). Among the 112 samples analysed at the CRL, only the positive or doubtful samples with one of the screening methods (PremißTest, FPT, STAR) were confirmed by LC/MS-MS (88 samples).

After the confirmatory analyses, 31 samples really contained antimicrobial residues $(35 \%$ of the confirmed samples) at different levels (26 samples at concentrations lower than the respective MRLs and 5 only at concentrations higher than the respective MRLs). 
401 The false positive rates of the FPT, the Premi®Test and the STAR protocol were $3 \%$ $402\left((1 /(1+31))^{*} 100\right), 62 \%\left((52 /(52+31))^{*} 100\right)$ and $31 \%\left((14 /(14+31))^{*} 100\right)$ respectively. The false 403 positive (rate of each method was calculated as the number of positive results for blank 404 samples (free of antibiotic substances) divided by the total number of positive samples (the same number plus the number of contaminated samples showing positive results) and multiplied by 100 .

52 samples were PremißTest positive or doubtful, but no antibiotic residue could be identified when analyzed by LC/MS-MS (). However, a false-positive result could be due to the degradation of the antimicrobials initially contained in the sample (too long delay before the analysis by LC/MS-MS, very unstable molecules....). Furthermore, it could be false compliant results of the confirmatory method. The antimicrobial present in the sample could not be detected and identified by the multi-residue LC/MS-MS method if the molecule is not present in the spectrum of detection of the method (metabolites, ...). or the sensitivity could be insufficient.

After a positive result with Premi®Test, the presence of antimicrobial residues was confirmed in 26 samples (33\%), from which 4 samples contained antimicrobials at concentrations upper than the MRL. Three of the 10 positive FPT samples and 4 of the STAR positive samples were at concentrations upper than the MRL. Premi®Test and STAR screened positive 22 and 13 samples respectively which really contained antimicrobial compounds at concentrations lower than MRL. The FPT detected only 6 of these samples. Therefore, the sensitivity of PremißTest and STAR protocol was lower than those of the FPT. The highest confirmatory rate was obtained for the FPT (90\%). The false negative rates of the FPT, the Premi®Test and the STAR protocol were equal to $8 \%, 28 \%$ and $20 \%$ respectively. The false negative rate was calculated as the number of false negative results divided by the total of the true negative samples plus the number of false negative results for each method. 
429 The most commonly detected antimicrobial classes detected during this pilot study were 430 beta-lactams (penicillin and amoxicillin) (15 samples) and tetracyclines (doxycycline, 431 tetracycline, oxytetracycline and chlortetracycline) (15 samples). Premi ${ }^{\circ}$ Test (12 positive 432 samples) was much more sensitive for the detection of beta-lactams than the FPT (1 positive sample) and the STAR protocol (5 positive samples), even too sensitive in some cases (10 positive samples confirmed at concentrations lower than MRL). Premi®Test was also more sensitive for the screening of sulphonamides than the 2 other methods (FPT and STAR failed to detect 1 sample at a concentration higher than the MRL). Finally, Premi ${ }^{\circledR}$ Test was less sensitive than the FPT and especially than the STAR protocol for the detection of tetracyclines. Therefore, this study demonstrated the ability of PremißTest to detect samples at the MRL level for sulphonamides and beta-lactams, but not for tetracyclines. These conclusions confirmed the results obtained by different teams during the past years ((Reybroeck 2000a, (Reybroeck 2000b, Okerman et al. 2004, Popelka et al. 2005). obtained with Premi®Test and 7 with FPT.

Table VII presented the combined results of a previous study realized in 2003 and 2004 (64 samples really containing antimicrobial residues) with the pilot study.

"[Insert Table VII about here]"

After the confirmatory analyses of positive samples, 31 samples contained antimicrobial residues at concentrations lower than the respective MRLs and 33 samples at concentrations higher than the respective MRLs. The total number of positive samples reported with Premi®Test (52) was higher than with FPT (38). However, the number of samples detected positive with Premi®Test (26) or FPT (25) which really contained antimicrobial compounds at concentrations higher than MRL was identical. Moreover, 6 false negative results were 
455 The presence of beta-lactams was confirmed in 17 samples by the LC/MS-MS method. All of 456 these 17 samples were detected positive or doubtful samples with Premi®Test, while only 7 457 samples were detected positive with the FPT ( $1<M R L$ and $6>M R L)$. Therefore, the detection 458 capability of Premi®Test for beta-lactams was better than FPT (). The sensitivity was also 459 lower for sulphonamides (4 positive samples PremißTest, 4 negative samples FPT). Two of 460 these samples really contained sulphonamides at concentrations higher than the MRL and 461 the FPT failed to detect them while the PremißTest succeeded to detect. However, the 462 sensitivity of FPT for tetracyclines was better (15 doubtful or positive samples with 463 PremißTest instead of 19 positive samples with FPT).

The most detected antimicrobials were tetracyclines (25 samples)and beta-lactams (17 samples). Then, macrolides (6), sulphonamides (4), quinolones (3) or mix of antimicrobials (8) were found.

\section{Collaborative study}

Results of the expert laboratory. The expert laboratory obtained only one positive false result for the 2 series of analyses for the white chicken sample (code 10), whereas the same sample (code 27) was found negative for the 2 series. The L1 concentration was selected to give negative results (Table I). The expert laboratory did not obtain any false negative result because the L3 concentration was that designed to give positive results, whereas the L2 concentration was to be at the limit of sensitivity of the test.

It should be noted that the later analysis of the results was carried out while taking as principle that the doubtful results are positive results, this which is applied at the time for routine analyses. 
482 The results of the expert laboratory were very satisfactory (Table VIII): 14 blank samples out 483 of 16 were negative (only 2 positive results for chicken samples) (L0). Below the assumed 484 limit of detection, 14 samples out of 16 were negative (L1). At the assumed limit of detection, 485 all the samples were positive (L2). Above the assumed limit of detection, all the samples were positive (L3).

The results were homogeneous between laboratories. The results were analysed and summarised in Table X. Concerning negative samples (L0) (16 by laboratories corresponding to 3 different species), 4 laboratories found $100 \%$ of negative results, 5 laboratories found 1 or 2 samples doubtful or positive, sometimes in only one series of analyses (laboratories M and $\mathrm{N})$.

\section{"[Insert Table IX about here]"}

"[Insert Table X about here]"

For the samples containing antibiotics at a concentration below the detection limit (L1), 6 laboratories found $100 \%$ of negative results; 3 laboratories found 1 or 2 samples which were doubtful or positive. When the concentration was considered near the detection limits (L2), 
510 tylosin was generally detected (83 \% of positive results), whereas the other antibiotics were

511 less detected (oxytetracycline 11\%, ceftiofur $11 \%$ and sulfamethazine $17 \%$ ).

The average rate of positive samples to the L3 concentration was $81 \%$. The rate of detection to the $L 3$ concentration is $100 \%$ for the 4 antibiotics and for 3 laboratories $(B, D, N)$. This rate was $100 \%$ for the totality of the laboratories concerning tylosin and ceftiofur.

The results of the participating laboratories were analyzed in order to calculate different validation parameters. A laboratory was removed from the analysis because due to a delay in transport the samples arrived in defrosted conditions.

Table X presents also the analysis of the results of all the participating laboratories in term of reproducibility, by material (combination animal/antibiotic species) and in a global way. The reproducibility, expressed as a percentage, is the ratio of the number of identical results, the most common type (e.g. negative results for blank samples or positive result for samples contaminated with an antimicrobial concentration exceeding the detection limit) on the total number of analyses.

The results of the participating laboratories in term of reproducibility are very satisfactory with an average percentage of $89,1 \%$. The worse reproducibility was observed for the combination bovine/sulfamethazine. A little higher concentration of sulfamethazine would undoubtedly have given better results in term of reproducibility.

The percentage of specificity SP for the levels L0 and L1 was calculated as: SP $=\left[1(\mathrm{FP} / \mathrm{N}-)^{*}\right.$ 100\%] where: N-: total number of tests L0 and L1 and FP: number of false positive results. The percentage of sensitivity SE for each positive contamination level L2 and L3 was 536 calculated using the following equation: $\left.\mathrm{SP}=\left(\mathrm{TP} / \mathrm{N}_{+}\right)\right)^{*} 100 \%$ where: $\mathrm{N}+$ : total number of tests L2 or L3 respectively and TP: a number of true positive. 
538 Specificity $(95,3 \%)$ and sensitivity of the Premi®Test to the level L3 $(72,5 \%)$ were very satisfactory.

The repeatability was estimated in each laboratory: 1 - by comparing the results of the 2 tests performed on each sample (2 different sets of analyses), knowing that the knowledge of the initial result can influence the reading at the second analysis, 2- by comparing the results obtained with the 2 samples of each pair. The repeatability expressed as a percentage, is the ratio of the number of identical results per couple of analyses on the total number of couples. The following table presents the total analysis of the results of all the participants obtained with the Premi®Test in term of repeatability. (Tables XI).

"[Insert Table XI about here]"

The results of the participating laboratories in term of repeatability are very satisfactory with an average percentage of $94,8 \%$ for the same sample and $92,7 \%$ per 2 identical samples (pair). The limits of detection of the Premi®Test during the preliminary study were confirmed by the collaborative study.

\section{Conclusion}

This paper presents a significant dataset concerning the performance of the Premi®Test, for different kind of samples: spiked meat juice samples, incurred samples, routine field samples (confirmed by a LC/MS-MS method). The detection capabilities of Premi®Test for betalactams (amoxicillin, ceftiofur), one macrolide (tylosin) and tetracycline were at the level of the respective Maximum Residue Limits (MRL) in muscle samples or even lower. The applicability of the test to different animal species was proven. Moreover, the applicability of Premi ${ }^{\circ}$ Test to routine analysis of samples was demonstrated. The Four Plate Test (reference method) and the PremißTest showed comparable performances in term of sensitivity and specificity. Moreover, the false negative rate of PremißTest was always lower than that of the FPT. This is the most important parameter to minimise for a screening 
566 method for antibiotic residues. On the contrary, the false positive rate of Premi®Test 567 appeared, in step 3, higher than that of the FPT. This means that the number of samples to confirm by physico-chemical methods would be higher if the laboratories used the Premi®Test alone. However, it means also that more real positive samples would be detected because Premi®Test was more sensitive than FPT for some antimicrobials (betalactams and sulphonamides). Beta-lactams and some sulphonamides were satisfactorily detected at the MRL level by the Premi®Test.

This is the first time to our knowledge that an interlaboratory study organised for the detection of antibiotics with Premi®Test was described. The results of the collaborative study were very satisfactory. The results of 9 laboratories were finally analyzed, plus the expert laboratory. Specificity was estimated at $95.3 \%$, moreover the sensitivity of the test to the L3 level was calculated to $72,5 \%$. These results were similar with those obtained at the time of preceding validations of kits of detection of the antibiotic residues in milk. The results in term of repeatability and reproducibility are very satisfactory, with average percentages of $94,8 \%$ and $92,7 \%$ for the repeatability and of 89,1 for reproducibility.

In conclusion, Premi®Test is easy to perform. It is ideal for an "on site" use (slaughterhouses, test laboratories) as no special laboratory equipment is needed to perform the test. The rapidly response "yes/no" result is simply read by colour comparison. Premi®Test is applicable to the muscles of various species (porcine, bovine, ovine...), by using as negative control a "blank" muscle of each analyzed specie to optimise the reading time.

Finally, since 2006, the field laboratories in France are authorised to use the PremißTest as a pre-screening test. All positive samples with Premi®Test are then mandatory analysed by the FPT (Anon. 2006). All positive samples with the FPT are sent to our laboratory for confirmation as usual. 


\section{Acknowledgements}

595 The authors thank Annie Rault, Anne de Courville, Sophie Gautier and Marie-Pierre 596 Fourmond for their technical participation to the preliminary study and Catherine Creff-Froger 597 responsible of the Four Plate Test analyses. We would like to thank also Jean-Michel Fabre 598 (Phylum, Labège, France) for its help to the data analysis. Thanks to Françoise Goeijen 599 (DSM, The Netherlands), and to the 6 French field veterinary laboratories (LVD) which 600 participated to the preliminary study. Thanks finally to all the participants of the collaborative 601 study.

602

603

604 


\section{References}

ANON., 2005a, AFNOR Document, "Requirements relating to the preliminary and collaborative studies undertaken by an expert laboratory/application to the detection methods of antimicrobial residues and another molecules for related purpose" revision 0.

ANON., 2005b, Memorandum DGAL/SDRRCC/N2005-8217 of the 08/09/2005, «New method of analysis for the research of antimicrobial residues in meat with Premi ${ } T e s t$ and Bacillus cereus: implementation of a pilot action ». http://agriculture.gouv.fr/

ANON., 2006, Memorandum DGAL/SDRRCC/N2006-8240 of the 04/10/2006, « Control plan for antimicrobial residues in slaughter meats, poultry, game, rabbits and breeding fish ». $\underline{\text { http://agriculture.gouv.fr/ }}$

ARTS, C. J. M., GEIJP, E., STARK, J., WITKAMP, R., 2000, Premi®Test: a broad spectrum screening test for detection of antimicrobial compounds in meat, organs and urine. In: Proceedings of the EuroResidue IV Conference (Veldhoven, The Netherlands), Van Ginkel LA, Ruiter A., 186-192.

ARTS, C. J. M. and WITKAMP, R. F., 1999, The Premi Test for screening for residues of antimicrobial compounds in meat, A summary report. TNO nutrition and Food Research Institute, TNO project number 50.736/01.01 (The Netherlands).

BOGAERTS, R., WOLF, F., 1980, A standardized method for the detection of residues of antibacterial substances in fresh meat. Die Fleischwirtschaft, 60, 672-674.

CANTWELL, H. and O'KEEFFE, M. 2006, Evaluation of the Premi®Test and comparison with the One Plate Test for the detection of antimicrobial substances in kidney. Food Additives and Contaminants, 23 (2), 120-125.

Council Regulation (EEC) No. 2377/90. 26 June 1990. Laying down a Community procedure for the establishment of maximum residue limits of veterinary medicinal products in foodstuffs of animal origin.

FABRE, J.M., 2003, Des Méthodes de recherche d'antibiotiques dans la viande se développent en France. La Semaine Vétérinaire, July 2003. 
632 FABRE, J.M., 2003, Recherche des résidus d'antibiotiques dans la viande: De nouvelles méthodes pour de nouveaux besoins. La Semaine Vétérinaire July 2003

FABRE, J.M., MIRCOVICH, C., GEIJP, E., 2004, Antibiotic residues in pork and poultry meat in France: the current situation and an evaluation of a new screening test. Bulletins des GTV, 23, 305-309.

GAUDIN, V., MARIS, P., FUSELIER, R., RIBOUCHON, J.L., CADIEU, N., RAULT, A., 2004, Validation of a microbiological method: the STAR protocol, a five-plate test, for the screening of antibiotic residues in milk. Food Additives and Contaminants, 21, 422-433.

OKERMAN, L., CROUBELS, S., CHERLET, M., DE WASCH, K., DE BACKER, P., VAN HOOF, J., 2004, Evaluation and establishing the performance of different screening tests for tetracycline residues in animal tissues. Food Additives and Contaminants, 21, 145153.

POPELKA, P., NAGY, J., GERMUSKA, R., MARCINCAK, S., JEVINOVA, P., DE RIJK, A., 2005 , Comparison of various assays used for detection of beta-lactam antibiotics in poultry meat. Food Additives and Contaminants, 22, 557-562.

REYBROEK, W., 2000a, Performance of the Premi®Test using naturally contaminated meat. In: Proceedings of the EuroResidue IV Conference (Veldhoven, The Netherlands), Van Ginkel LA, Ruiter A., 909-912.

REYBROEK, W., 2000b, Detection of residues of antibiotics in foodstuffs with microbiological tests using bacillus. Proceedings of the Bacillus symposium; 2000 August 30-31; Brugge, Belgium.

STEAD, S., SHARMAN, M., TARBIN, J.A., GIBSON, E., RICHMOND, S., STARK, J., GEIJP, E., 2004, Meeting maximum residue limits: an improved screening technique for the rapid detection of antimicrobial residues in animal food products. Food Additives and Contaminants, 21, 216-221. 
661

662

663

664

665

666

667

668

669

670 
1 Table I. Interlaboratory study: Content of the $\mathbf{1 6}$ materials sent in blind duplicate.

\begin{tabular}{cccccc}
\hline & & Porcine & Bovine & Porcine & Chicken \\
\hline \multirow{2}{*}{$\begin{array}{c}\text { Antimicrobial } \\
\end{array}$} & $\begin{array}{c}\text { Level of } \\
\text { contamination }\end{array}$ & Oxytetracycline & Sulfamethazine & Ceftiofur & Tylosin \\
\hline MRL & $/$ & 100 & 100 & 1000 & 100 \\
\hline \multirow{2}{*}{\begin{tabular}{c} 
Spiked \\
concentrations \\
\cline { 2 - 6 }$(\mu \mathrm{g} / \mathrm{kg})$
\end{tabular}} & L0 & 'Blank' & 'Blank' & 'Blank' & 'Blank' \\
\cline { 2 - 6 } & L2 & 20 & 20 & 40 & 10 \\
\hline
\end{tabular}


Table II. Results of the Premi®Test analyses on spiked juice samples (step 1). 5

\begin{tabular}{|c|c|c|c|c|c|c|c|}
\hline Antibiotic family & SULPHONAMIDE & TETRACYCLINE & MACROLIDE & $\begin{array}{c}\text { BETA- } \\
\text { LACTAM }\end{array}$ & $\begin{array}{l}\text { AMINOGLYC } \\
\text { OSIDE }\end{array}$ & $\begin{array}{c}\text { BETA- } \\
\text { LACTAM }\end{array}$ & \\
\hline Antibiotic & Sulfamethazine & Oxytetracycline & Tylosin & Amoxicillin & Gentamicin & Ceftiofur & Global \\
\hline $\begin{array}{l}\text { Tested concentrations }(\mu \mathrm{g} \\
\left.\qquad \mathrm{kg}^{-1}\right)\end{array}$ & $50 / 100 / 200$ & $50 / 100 / 200$ & $50 / 100 / 200$ & $25 / 50 / 100$ & $50 / 100 / 200$ & $100 / 200 / 400$ & l \\
\hline Detection rate at MRL & $20 \%$ & $80 \%$ & $100 \%$ & $100 \%$ & $0 \%$ & $(100 \%)$ & $67 \%$ \\
\hline Detection rate at $2^{*} \mathrm{MRL}$ & $100 \%$ & $100 \%$ & $100 \%$ & $100 \%$ & $40 \%$ & $(100 \%)$ & $90 \%$ \\
\hline Detection capability & 2xMRL & 2xMRL & MRL & $0.5 \times M R L$ & $>2 \times M R L$ & $0.5 \times M R L$ & I \\
\hline
\end{tabular}

$7 \quad{ }^{*} M R L$ in bold character

8

9 * The percentage is between brackets when there were no analyses performed at this level of 10 concentration. i.e. All the tested concentrations for ceftiofur were below MRL and even 0.5 MRL. At

$11400 \mathrm{~g} \mathrm{~kg}^{-1}, 100 \%$ of the results were positive or doubtful. 
12 Table III. Analyses of naturally incurred samples with the 3 screening methods (step 2).

13

\begin{tabular}{|c|c|c|c|c|c|c|c|}
\hline Antibiotic & OTC/SDMX & Amoxicillin & Tylosin & $\begin{array}{l}\text { Global results of } \\
\text { incurred samples }\end{array}$ & Blank & $\mathrm{Fp}+$ & $\mathrm{Fp}-$ \\
\hline $\begin{array}{c}\text { MRL (pig muscle) } \\
(\mu \mathrm{g} / \mathrm{kg})\end{array}$ & $100 / 100$ & 50 & 100 & & & & \\
\hline $\begin{array}{l}\text { Concentrations by } \\
\text { LC/MS-MS }(\mu \mathrm{g} / \mathrm{kg})\end{array}$ & $760 / 150$ & 270 & 750 & & & & \\
\hline $\begin{array}{l}\text { Number of positive } \\
\text { results with } \\
\text { Premi }{ }^{\circledR} \text { Test }\end{array}$ & $5 / 5$ & $5 / 5$ & $5 / 5$ & $15 / 15$ & $0 / 5$ & $0 \%$ & $0 \%$ \\
\hline $\begin{array}{l}\text { Number of positive } \\
\text { results with STAR }\end{array}$ & $5 / 5$ & $5 / 5$ & $5 / 5$ & $15 / 15$ & $3 / 5$ & $60 \%$ & $0 \%$ \\
\hline $\begin{array}{l}\text { Number of positive } \\
\text { results with FPT }\end{array}$ & $5 / 5$ & $4 / 5$ & $1 / 5$ & $10 / 15$ & $2 / 5$ & $40 \%$ & $33 \%$ \\
\hline
\end{tabular}

14 Fp+: false positive rate; fp-: false negative rate; OTC: Oxytetracycline; SDMX: sulfadimethoxine 
16 Table IV. Correlation between field laboratories and AFSSA, comparison of the 3 screening

17 tests and LC/MS-MS method in relation to the species (step 3). 18

\begin{tabular}{|c|c|c|c|c|c|c|c|}
\hline & Specie & Bovine & Porcine & Poultry & Others & Unknown & TOTAL \\
\hline \multirow[b]{2}{*}{ LVD } & $\begin{array}{c}\text { Number of samples analysed } \\
\text { at } L V D^{\mathrm{b}}\end{array}$ & 379 & 671 & 205 & 26 & 146 & 1427 \\
\hline & $\begin{array}{c}\text { Number of positive and } \\
\text { doubtful results at LVD } \\
\text { Premi®Test }\end{array}$ & 63 & 26 & 1 & 5 & 7 & 102 \\
\hline \multirow{5}{*}{ AFSSA } & $\begin{array}{c}\text { Number of samples analysed } \\
\text { at AFSSA }\end{array}$ & 65 & 31 & 4 & 5 & 7 & $112^{a}$ \\
\hline & $\begin{array}{c}\text { Number of positive and } \\
\text { doubtful results with } \\
\text { Premi®Test }\end{array}$ & 45 & 19 & 2 & 5 & 7 & 78 \\
\hline & Correlation LVD/AFSSA (\%) & 69 & 61 & 50 & 100 & 100 & 76 \\
\hline & $\begin{array}{l}\text { Number of positive samples } \\
\text { with FPT }\end{array}$ & 3 & 5 & 2 & 0 & 0 & 10 \\
\hline & $\begin{array}{c}\text { Number of positive samples } \\
\text { with STAR }\end{array}$ & 13 & 13 & 4 & 0 & 2 & 32 \\
\hline \multirow{3}{*}{$\begin{array}{l}\text { AFSSA } \\
\text { LC/MS- } \\
\text { MS }\end{array}$} & Number of tested samples & 38 & 18 & 4 & 1 & 0 & 61 \\
\hline & $\begin{array}{l}\text { Number of positive samples } \\
\text { (identified molecule) }\end{array}$ & 14 & 8 & 3 & 0 & 0 & 25 \\
\hline & $\begin{array}{l}\text { Rate of positive confirmation } \\
(\%)\end{array}$ & 37 & 44 & 75 & 0 & I & 41 \\
\hline
\end{tabular}

19

${ }^{a}$ Total of the samples sent to AFSSA for confirmation

${ }^{b}$ LVD means "Laboratoire Vétérinaire Départemental" in French which is a field veterinary laboratory. 
23 Table V. Correlation between the PremißTest and the FPT (step 3).

\begin{tabular}{ccc}
\hline & $\begin{array}{c}\text { 1st analysis (112 } \\
\text { samples) }\end{array}$ & $2^{\text {nd }}$ analysis (1427 \\
& & samples) \\
\hline Relative accuracy AC (\%) & 33.9 & 94.8 \\
\hline Relative specificity SP (\%) & 30.4 & 95.0 \\
\hline Relative sensitivity SE (\%) & 70.0 & 70.0 \\
\hline
\end{tabular}

24 Relative accuracy: $A C=(P A+N A) / N^{*} 100 \%$

25 Relative specificity: $S P=(N A / N-)^{*} 100 \%$

26 Relative sensitivity: $S E=(P A / N+) * 100 \%$

27 Where: NA is the negative agreement, PA: the positive agreement, ND: the negative discrepancy, PD: the 28 positive discrepancy;

$29 N=N A+P a+P D+N D$ : total number of samples

$30 \quad \mathrm{~N}$ - is the total number of negative samples obtained with the reference method $(N A+P D)$

$31 N+$ is the total number of positive samples obtained with the reference method $(P A+N D)$ 
33 Table VI. Results of the identification of positive samples with LC/MS-MS at the AFSSA:

34 Comparison of Premi®Test, Four Plate Test and STAR protocol in 2005 (step 3).

\begin{tabular}{|c|c|c|c|c|c|c|c|c|c|}
\hline \multirow[t]{2}{*}{ Identified AB family } & \multirow{2}{*}{$\begin{array}{c}\text { Quantification / } \\
\text { MRL }\end{array}$} & \multirow{2}{*}{$\begin{array}{l}\text { Number of } \\
\text { analysed }\end{array}$} & \multicolumn{3}{|c|}{ PremißTest results } & \multicolumn{2}{|c|}{ FPT results } & \multicolumn{2}{|c|}{ STAR results } \\
\hline & & & - & D & + & - & + & - & + \\
\hline MACRO & $<M R L$ & 1 & & 1 & & & 1 & & 1 \\
\hline SULFA & $<M R L$ & 1 & & 1 & & 1 & & 1 & \\
\hline TTC & $<M R L$ & 10 & 4 & 3 & 3 & 6 & 4 & 2 & 8 \\
\hline TTC & $>\mathrm{MRL}$ & 2 & 1 & & 1 & & 2 & & 2 \\
\hline $\mathrm{BL}$ & $<\mathrm{MRL}$ & 10 & & 2 & 8 & 10 & & 7 & 3 \\
\hline $\mathrm{BL}$ & $>M R L$ & 2 & & & 2 & 1 & 1 & & 2 \\
\hline TTC + SULFA & $<M R L$ & 1 & & & 1 & & 1 & & 1 \\
\hline $\mathrm{BL}+$ sulfa & $>\mathrm{MRL}$ & 1 & & & 1 & 1 & & 1 & \\
\hline MACRO + TTC + BL & $<\mathrm{MRL}$ & 1 & & & 1 & 1 & & 1 & \\
\hline $\mathrm{TTC}+\mathrm{BL}$ & $<\mathrm{MRL}$ & 1 & & & 1 & 1 & & 1 & \\
\hline $\mathrm{AMINO}+\mathrm{BL}$ & $<M R L$ & 1 & & & 1 & 1 & & 1 & \\
\hline \multirow{3}{*}{ Global } & $<$ LMR & 26 & 4 & 7 & 15 & 20 & 6 & 13 & 13 \\
\hline & $>$ LMR & 5 & 1 & 0 & 4 & 2 & 3 & 1 & 4 \\
\hline & Total & 31 & 5 & 7 & 19 & 22 & 9 & 14 & 17 \\
\hline Absence & I & 57 & 5 & 8 & 44 & 56 & 1 & 43 & 14 \\
\hline Not analysed & l & 24 & 24 & & & 24 & & 24 & \\
\hline TOTAL & I & 112 & 34 & 15 & 63 & 102 & 10 & 81 & 31 \\
\hline
\end{tabular}


Table VII. Results of the identification of positive samples with LC/MS-MS at the AFSSA:

39 Comparison of alternative method (Premi®Test) and reference method (FPT) from 2003 to

402005 (step 3).

41

\begin{tabular}{|c|c|c|c|c|c|c|c|}
\hline \multirow[t]{2}{*}{ Identified AB family } & \multirow{2}{*}{$\begin{array}{c}\text { Quantification / } \\
\text { MRL }\end{array}$} & \multirow{2}{*}{$\begin{array}{c}\text { Number of } \\
\text { analysed samples }\end{array}$} & \multicolumn{3}{|c|}{ Premi®Test results } & \multicolumn{2}{|c|}{ FPT results } \\
\hline & & & - & $\mathbf{D}^{*}$ & + & - & + \\
\hline \multirow[t]{3}{*}{ Beta-lactams } & $<\mathrm{MRL}$ & 9 & l & 1 & 8 & 8 & 1 \\
\hline & $>\mathrm{MRL}$ & 8 & 1 & I & 8 & 2 & 6 \\
\hline & Sum ${ }^{\star \star \star}$ & 17 & & & & & \\
\hline \multirow[t]{3}{*}{ Tetracyclines } & $<\mathrm{MRL}$ & 13 & 5 & 4 & 4 & 6 & 8 \\
\hline & $>\mathrm{MRL}$ & 12 & 5 & 1 & 6 & I & 11 \\
\hline & Sum & 25 & & & & & \\
\hline \multirow[t]{3}{*}{ Sulphonamides } & $<M R L$ & 2 & l & 1 & 1 & 2 & I \\
\hline & $>\mathrm{MRL}$ & 2 & l & I & 2 & 2 & 1 \\
\hline & Sum & 4 & & & & & \\
\hline Quinolones & $>\mathrm{MRL}$ & 3 & 1 & I & 2 & I & 3 \\
\hline \multirow[t]{3}{*}{ BL + Sulfa } & $<\mathrm{MRL}$ & 2 & l & I & 2 & 1 & 1 \\
\hline & $>\mathrm{MRL}$ & 2 & l & l & 2 & 2 & 1 \\
\hline & Sum & 4 & & & & & \\
\hline \multirow[t]{3}{*}{ Tetra + Sulfa } & $<\mathrm{MRL}$ & 1 & l & I & 1 & I & 1 \\
\hline & $>\mathrm{MRL}$ & 3 & l & l & 3 & I & 3 \\
\hline & Sum & 4 & & & & & \\
\hline Tetra + BL & $<\mathrm{MRL}$ & 1 & l & I & 1 & 1 & l \\
\hline \multirow[t]{3}{*}{ Macrolides } & $<\mathrm{MRL}$ & 3 & 1 & 1 & 1 & 1 & 2 \\
\hline & $>\mathrm{MRL}$ & 3 & 1 & 1 & 3 & 1 & 2 \\
\hline & Sum & 6 & 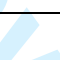 & & & & \\
\hline \multirow{2}{*}{ Global } & $<$ MRL & 31 & 6 & 7 & 18 & 19 & 13 \\
\hline & $>$ MRL & 33 & 6 & 1 & 26 & 7 & 25 \\
\hline TOTAL & 1 & 64 & 12 & 8 & 44 & 26 & 38 \\
\hline
\end{tabular}

\section{*D: Doubtful}

${ }^{* *}$ MRL: Maximum Residue Limit

${ }^{* * \star}$ Sum of samples confirmed lower and higher than respective MRLs. 
Table VIII. Results of the expert laboratory (AFSSA) during the interlaboratory 47 study.

48

\begin{tabular}{ccccc}
\hline $\begin{array}{c}\text { Level of } \\
\text { contamination }\end{array}$ & $\begin{array}{c}\text { Oxytetracycline } \\
\text { Porcine }\end{array}$ & $\begin{array}{c}\text { Sulfamethazine } \\
\text { Porcine }\end{array}$ & $\begin{array}{c}\text { Ceftiofur } \\
\text { Bovine }\end{array}$ & $\begin{array}{c}\text { Tylosin } \\
\text { Chicken }\end{array}$ \\
\hline L0 & $0^{*}$ & 0 & 0 & 2 \\
\hline L1 & 0 & 0 & 0 & 2 \\
\hline L2 & 4 & 4 & 4 & 4 \\
\hline L3 & 4 & 4 & 4 & 4
\end{tabular}


51 Table IX. Raw data of the participants for each material and the $\mathbf{1 6}$ combinations.

\begin{tabular}{|c|c|c|c|c|c|c|c|c|c|c|c|c|c|c|c|c|}
\hline & \multicolumn{16}{|c|}{ Porcine Oxytetracycline } \\
\hline & \multicolumn{4}{|c|}{ LO (0) } & \multicolumn{4}{|c|}{ L1 $\left(20^{\star}\right)$} & \multicolumn{4}{|c|}{ L2 (200) } & \multicolumn{4}{|c|}{ L3 (400) } \\
\hline & \multicolumn{2}{|c|}{ Mat 1} & \multicolumn{2}{|c|}{ Mat 5} & \multicolumn{2}{|c|}{ Mat 2} & \multicolumn{2}{|c|}{ Mat 6} & \multicolumn{2}{|c|}{ Mat 3} & \multicolumn{2}{|c|}{ Mat 7} & \multicolumn{2}{|c|}{ Mat 4} & \multicolumn{2}{|c|}{ Mat 8} \\
\hline Lab & A1 & A2 & A1 & A2 & A1 & A2 & A1 & A2 & A1 & A2 & A1 & A2 & A1 & A2 & A1 & A2 \\
\hline $\mathbf{A}$ & - & - & - & - & - & - & - & - & - & - & - & - & - & - & - & $\mathrm{D}$ \\
\hline B & - & - & - & - & - & - & - & - & - & - & - & - & + & + & + & + \\
\hline $\mathbf{C}$ & - & - & - & - & - & - & - & - & - & - & - & - & + & + & + & + \\
\hline D & - & - & - & - & - & - & - & - & - & - & - & - & + & + & + & + \\
\hline$E$ & - & - & - & - & - & - & - & - & - & - & - & - & - & - & - & - \\
\hline$F$ & - & - & - & - & - & - & - & - & + & + & + & + & + & + & + & + \\
\hline $\mathbf{G}$ & - & - & - & - & - & - & - & - & - & - & - & - & D & $\mathrm{D}$ & D & D \\
\hline $\mathbf{M}$ & - & - & - & - & - & - & - & - & - & - & - & - & - & - & - & - \\
\hline $\mathbf{N}$ & - & - & - & - & - & - & - & - & - & - & - & - & + & + & + & + \\
\hline
\end{tabular}

Porcine ceftiofur

\begin{tabular}{|c|c|c|c|c|c|c|c|c|c|c|c|c|c|c|c|c|}
\hline & \multicolumn{4}{|c|}{ LO (0) } & \multicolumn{4}{|c|}{ L1 (40) } & \multicolumn{4}{|c|}{ L2 (400) } & \multicolumn{4}{|c|}{ L3 (800) } \\
\hline & \multicolumn{2}{|c|}{ Mat 9} & \multicolumn{2}{|c|}{ Mat 13} & \multicolumn{2}{|c|}{ Mat 10} & \multicolumn{2}{|c|}{ Mat 14} & \multicolumn{2}{|c|}{ Mat 11} & \multicolumn{2}{|c|}{ Mat 15} & \multicolumn{2}{|c|}{ Mat 12} & \multicolumn{2}{|c|}{ Mat 16} \\
\hline Lab & A1 & A2 & A1 & A2 & A1 & A2 & A1 & A2 & A1 & A2 & A1 & A2 & A1 & A2 & A1 & A2 \\
\hline A & - & - & - & - & - & - & - & - & - & $\mathrm{D}$ & - & - & + & $\mathrm{D}$ & + & + \\
\hline B & - & - & - & - & - & - & - & - & - & - & - & - & + & + & + & + \\
\hline C & - & - & + & + & - & - & - & - & - & - & - & - & + & + & + & + \\
\hline D & - & - & - & - & - & - & - & - & - & - & - & - & + & + & + & + \\
\hline$E$ & - & - & - & - & - & - & - & - & - & - & - & - & $\mathrm{D}$ & $\mathrm{D}$ & + & + \\
\hline $\mathbf{F}$ & $\mathrm{D}$ & $\mathrm{D}$ & $\mathrm{D}$ & $\mathrm{D}$ & - & - & $\mathrm{D}$ & $D$ & - & $\mathrm{D}$ & + & + & + & + & + & + \\
\hline $\mathbf{G}$ & - & - & - & - & - & - & - & - & - & - & - & - & + & + & + & + \\
\hline $\mathbf{M}$ & - & - & - & - & - & - & - & - & - & - & - & - & + & + & + & + \\
\hline $\mathbf{N}$ & - & - & - & - & - & - & - & - & - & - & - & - & + & + & + & + \\
\hline
\end{tabular}

Bovine sulfamethazine

\begin{tabular}{|c|c|c|c|c|c|c|c|c|c|c|c|c|c|c|c|c|}
\hline & \multicolumn{4}{|c|}{ Lo (0) } & \multicolumn{4}{|c|}{ L1 (20) } & \multicolumn{4}{|c|}{ L2 (200) } & \multicolumn{4}{|c|}{ L3 (400) } \\
\hline & \multicolumn{2}{|c|}{ Mat 17} & \multicolumn{2}{|c|}{ Mat 21} & \multicolumn{2}{|c|}{ Mat 18} & \multicolumn{2}{|c|}{ Mat 22} & \multicolumn{2}{|c|}{ Mat 19} & \multicolumn{2}{|c|}{ Mat 23} & \multicolumn{2}{|c|}{ Mat 20} & \multicolumn{2}{|c|}{ Mat 24} \\
\hline Lab & A1 & A2 & A1 & A2 & A1 & A2 & A1 & A2 & A1 & A2 & A1 & A2 & A1 & A2 & A1 & A2 \\
\hline A & - & - & - & - & - & - & - & - & - & - & - & - & $\mathrm{D}$ & - & - & - \\
\hline B & - & - & - & - & - & - & - & - & - & - & - & - & + & + & + & + \\
\hline C & - & - & - & - & - & - & - & - & - & - & - & - & - & - & - & - \\
\hline D & - & - & - & - & - & - & - & - & - & $\mathrm{D}$ & - & $\mathrm{D}$ & + & + & + & + \\
\hline$E$ & - & + & - & + & - & + & - & + & - & + & - & + & - & + & - & + \\
\hline $\mathbf{F}$ & - & - & - & - & - & - & - & - & - & - & + & + & - & - & $\mathrm{D}$ & D \\
\hline $\mathbf{G}$ & - & - & - & - & - & - & - & - & - & - & - & - & - & - & $D$ & $\mathrm{D}$ \\
\hline $\mathbf{M}$ & - & $\mathrm{D}$ & - & - & - & - & - & $\mathrm{D}$ & - & - & - & - & - & - & - & - \\
\hline $\mathbf{N}$ & + & - & - & - & - & - & - & - & - & - & - & - & $D$ & + & $\mathrm{D}$ & + \\
\hline
\end{tabular}

\section{Chicken Tylosin}

\begin{tabular}{ccccccccccccccccc}
\hline Conc & \multicolumn{4}{c}{ L0 $(0)$} & \multicolumn{4}{c}{ L1 $(10)$} & \multicolumn{4}{c}{ L2 $(100)$} & \multicolumn{4}{c}{ L3 $(200)$} \\
\hline & \multicolumn{1}{c}{ Mat 25 } & \multicolumn{2}{c}{ Mat 29 } & \multicolumn{2}{c}{ Mat 26 } & \multicolumn{2}{c}{ Mat 30 } & Mat 27 & Mat 31 & Mat 28 & Mat 32 \\
\hline Lab & A1 & A2 & A1 & A2 & A1 & A2 & A1 & A2 & A1 & A2 & A1 & A2 & A1 & A2 & A1 & A2 \\
\hline A & - & - & - & - & - & - & - & - & + & D & - & D & D & + & D & + \\
\hline B & - & - & - & - & - & - & - & - & + & + & + & + & + & + & + & + \\
\hline C & - & - & - & - & - & - & - & - & + & + & + & + & + & + & + & + \\
\hline D & - & - & - & - & - & - & - & - & + & + & + & + & + & + & + & + \\
\hline E & - & - & - & - & - & - & - & - & - & - & - & D & + & + & + & + \\
\hline F & - & - & - & - & - & - & - & - & + & + & + & + & + & + & + & + \\
\hline G & - & - & - & - & - & - & - & - & + & + & - & D & + & + & + & + \\
\hline M & - & - & - & - & - & - & - & - & D & - & D & D & + & + & + & + \\
\hline N & - & - & - & - & - & - & - & - & D & + & + & + & + & + & + & +
\end{tabular}

52 A1: first analysis; A2: second analysis; Mat: Material; *Conc: concentrations $\left(\mathrm{ng} \mathrm{m}^{1}{ }^{1}\right.$ ). 
Table X. Analysis of the results of all the participating laboratories in term of percentages of positive results for each material and in term of reproducibility, by material (combination animal/antibiotic species) and global reproducibility.

\begin{tabular}{|c|c|c|c|c|c|}
\hline Material & $\begin{array}{l}\text { Number of } \\
\text { sample } \\
\text { (number of } \\
\text { the pair) }\end{array}$ & $\begin{array}{l}\text { Level of } \\
\text { contamination }\end{array}$ & $\begin{array}{l}\text { False positive } \\
\text { and true } \\
\text { positive rates }\end{array}$ & $\begin{array}{l}\text { Percentages of } \\
\text { positive results } \\
\text { for each } \\
\text { material }\end{array}$ & $\begin{array}{l}\text { Reproducibility } \\
\text { (\%) }\end{array}$ \\
\hline \multirow{4}{*}{ Porcine OTC } & 1 & LO & $\mathrm{FPO} a)^{*}$ & $0 \%$ & 100.0 \\
\hline & 2 & L1 & FP1 b) & $0 \%$ & 100.0 \\
\hline & 3 & L2 & TP2 c) & $11 \%{ }^{* *}$ & $88.9^{* \star *}$ \\
\hline & 4 & L3 & TP3 d) & $69 \%$ & 69.4 \\
\hline \multirow{4}{*}{$\begin{array}{l}\text { Porcine } \\
\text { ceftiofur }\end{array}$} & 5 & Lo & FP0 a) & $11 \%$ & 83.3 \\
\hline & 6 & L1 & FP1 b) & $8 \%$ & 94.4 \\
\hline & 7 & L2 & TP2 c) & $17 \%$ & 88.9 \\
\hline & 8 & L3 & TP3 d) & $53 \%$ & 100.0 \\
\hline \multirow{4}{*}{$\begin{array}{c}\text { Bovine } \\
\text { sulfamethazine }\end{array}$} & 9 & LO & FP0 a) & $17 \%$ & 91.7 \\
\hline & 10 & L1 & FP1 b) & $6 \%$ & 91.7 \\
\hline & 11 & L2 & TP2 c) & $11 \%$ & 83.3 \\
\hline & 12 & L3 & TP3 d) & $100 \%$ & 52.8 \\
\hline \multirow{4}{*}{$\begin{array}{l}\text { Chicken } \\
\text { Tylosin }\end{array}$} & 13 & LO & FP0 a) & $0 \%$ & 100.0 \\
\hline & 14 & L1 & FP1 b) & $0 \%$ & 100.0 \\
\hline & 15 & L2 & TP2 c) & $83 \%$ & 83.3 \\
\hline & 16 & L3 & TP3 d) & $100 \%$ & 100.0 \\
\hline
\end{tabular}

TP : True positive rate

a) False positive at level $L_{0}$

b) False positive at level $L_{1}$

c) True positive at level $L_{2}$

d) True positive at level $L_{3}$

${ }^{*}$ At each level of contamination, 9 laboratories reported 4 results for 2 materials in blind duplicate. The total number of samples per level is $9 * 4=36$ samples.

** TP2 c) $=4$ TP / 36 samples at level $L_{2} * 100=11 \%$

${ }^{* * *}$ Reproducibility at $L_{2}=32$ negative results divided by 36 samples ${ }^{*} 100=88.9 \%$ 
69

70

71

72

Table XI. Global analysis of the results of all the participants in term of repeatability of the analyses with the Premi®Test.

\begin{tabular}{ccc}
\hline Lab & $\begin{array}{c}\text { (Number of identical results for the } \\
\text { same sample } / \mathrm{N})^{*} 100\end{array}$ & $\begin{array}{c}\text { (Number of identical results for } \mathbf{2} \\
\text { identical samples (pair) / N)*100 }\end{array}$ \\
\hline A & 87.5 & 87.5 \\
\hline B & 100.0 & 100.0 \\
\hline C & 100.0 & 93.8 \\
\hline D & 93.8 & 100.0 \\
\hline E & 71.9 & 96.9 \\
\hline F & 96.9 & 78.1 \\
\hline G & 96.9 & 90.6 \\
\hline M & 90.6 & 90.6 \\
\hline N & 96.9 & 96.9 \\
\hline Total & 94.8 & 92.7 \\
\hline
\end{tabular}

$N$ : total number of samples (32) 